\title{
LAS VARIACIONES GEOGRÁFICAS DE LA PERCEPCIÓN DE LA AMENAZA DEL CRIMEN ORGANIZADO Y EL NARCOTRÁFICO EN MÉXICO
}

Carlos J. Vilalta

BOUNDARY, n. In political geography, an imaginary line between two nations, separating the imaginary rights of one from the imaginary rights of the other.

Ambrose Pierce, 1911.

\section{INTRODUCGIÓN}

LA VARIABLE DEPENDIENTE EN ESTE ESTUDIO es la percepción de la amenaza del narcotráfico y el crimen organizado en México, y la variable independiente la geografía o contexto local. Elijo estudiar esta variable dependiente por dos motivos sustantivos y otro metodológico. El primer motivo sustantivo es que tanto el narcotráfico como el crimen organizado son un problema de seguridad pública y nacional de una magnitud enorme. El segundo motivo es que la mayor parte de las discusiones públicas en este respecto son, desafortunadamente, anecdóticas y a veces inclusive dogmáticas. Este estudio hace exactamente lo contrario. Finalmente, el beneficio metodológico es que la encuesta de México, las Américas y el Mundo contiene, para una diversidad de años, el mismo reactivo al respecto de la percepción de la ciudadanía en este tema y, además, ofrece al investigador un diseño muestral con (casi) los mismos estratos 
regionales todos los años; es decir, permite realizar comparaciones repetidas del mismo reactivo en años diferentes y entre regiones diferentes del país. ${ }^{1}$

El hallazgo principal es que el contexto local o el lugar en donde reside el individuo es el correlativo que, entre 2008 y 2012 en México, más fuertemente se asoció con la percepción sobre la amenaza que representan el narcotráfico y el crimen organizado. En la presencia de otros correlativos de tipo demográfico y socioeconómico individuales, el contexto local, o regional en este caso, fue la variable que mejor predijo la opinión del individuo en este asunto de seguridad pública. La prueba repetida de la hipótesis durante los años para los cuales contamos con información, le dan solidez a estos hallazgos.

A continuación este estudio se divide en cinco partes. Primero presento el marco teórico del estudio y fijo el debate académico y sus implicaciones en términos precisos. Posteriormente planteo el caso de estudio, en donde describo con ayuda de estadísticas oficiales el problema y el reto del narcotráfico y el crimen organizado. Esta parte sirve para ubicar al lector frente a la magnitud del problema de seguridad pública que enfrentamos. La tercera parte contiene la hipótesis y la metodología. La cuarta parte presenta los resultados del análisis. Y la quinta y última parte contiene una discusión detallada y propositiva de los resultados con miras a siguientes estudios con base en la encuesta de México, las Américas y el Mundo. ${ }^{2}$

\section{MARCo teórico}

Esta parte contiene dos discusiones: sobre las diferencias entre los efectos composicionales y los contextuales y sobre la diferencia entre lugar y espacio.

\footnotetext{
${ }^{1}$ Con algunas salvedades que se mencionan en la sección de metodología.

2 Para mayor información del proyecto, visite: http://www.lasamericasyelmundo.cide.edu
} 


\section{1 ¿Efectos composicional o contextuales?}

En el debate sólo hay dos posiciones y son totalmente contrarias: la geografía importa o no importa para determinar la percepción individual. Cada posición tiene sus propias premisas e implicaciones teóricas. ${ }^{3}$ Por un lado, si la geografía no importa, entonces las variaciones en la percepción tienen que ser un "efecto composicional" de la población residente en cada lugar. Por otro lado, si la geografía importa, entonces la variación en la percepción es resultado de un "efecto contextual" de cada lugar en los individuos residentes en tales lugares independiente de su composición. Aquí por "efecto composicional" se entiende el efecto dado por el conjunto de características demográficas y socioeconómicas de un grupo o población en un lugar, mientras que por "efecto contextual" nos referimos al resultado de la interacción entre el individuo y su medio "con la naturaleza del medio ambiente siendo construido a través del tiempo por los mismos actores". ${ }^{4}$ Estos efectos están basados en marcos teóricos diferentes y poseen implicaciones teóricas opuestas.

El llamado "efecto composicional" consiste en la idea de que la variación espacial del comportamiento, la percepción y la opinión es efecto del conjunto agregado de las características demográficas y socioeconómicas que covarían con la localización de los grupos o poblaciones humanas. Es decir, las diferencias regionales de comportamiento, percepción y opinión, son fundamentalmente consecuencia (y reflejo geográfico) de las diferencias demográficas y socioeconómicas también regionales. Por lo anterior la premisa teórica acerca de las causas de la opinión, desde esta perspectiva, es que lo que más importa es la "composición" del lugar y no el "contexto" o conjunto de interacciones sociales entre individuos en

${ }^{3} \mathrm{Si}$ el estudio del cambio lo debemos a Heráclito, el análisis del tiempo lo debemos a San Agustín, quien lo mencionó repetidamente en sus Confesiones. Comienzo con estas referencias porque todo, incluida la opinión de los individuos, es por su propia naturaleza cambiante.

${ }^{4}$ Colin Flint, "Forming Electorates, Forging Spaces: The Nazi Party Vote and the Social Construction of Space”, American Behavioral Scientist, vol. 41, núm. 9, 1998, p. 1292. 
cada lugar. Esta idea básicamente anti-geográfica deriva de un estudio clásico de McAllister y Studiar sobre el electorado británico en el que mostraron evidencia de que el voto regional en esa nación era en realidad "una división más funcional que territorial"; ${ }^{5}$ es decir, el voto regional como reflejo de un voto de "clase social" más que de "cultura local".

La implicación teórica es que, en lugares con población composicionalmente similar, se opinará también de forma similar, independientemente de su contexto local. Esta implicación, a su vez, es la base lógica de la llamada teoría de la Nacionalización, la cual tiene una fuerte relación con la teoría de la Modernización. Ambas teorías, Nacionalización y Modernización, comparten el supuesto de que el "lugar" o "contexto local" sí es una variable explicativa útil del comportamiento político, pero solamente en sociedades tradicionales, pequeñas y limitativas, y no en sociedades modernas y más urbanizadas. ${ }^{6} \mathrm{El}$ argumento de la nacionalización es que los procesos de modernización y urbanización tienden a homogeneizar ideas y opiniones, dejando la distancia geográfica entre los individuos y grupos sin mayor importancia para estos efectos de conformación de la opinión. En pocas palabras, no importa la localización, lo que importa es la conectividad. En consecuencia, la teoría de la Nacionalización establece que individuos similares en sus circunstancias demográficas y socioeconómicas, independientemente de su localización, tendrán la misma opinión sobre los mismos eventos. O dicho de otra manera, después de considerar tales circunstancias composicionales, las tendencias nacionales serán representativas de las tendencias regionales.

La hipótesis alternativa es totalmente contraria a lo anterior. Es aquella en que la geografía sí importa, más allá de las variables composicionales de la población en cada lugar. Aquí el argumento es que la población residente en un lugar (ej. colonia, ciudad, estado,

${ }^{5}$ Ian McAllister y Donley Studlar, "Region and Voting in Britain, 1979-87: Territorial Polarization or Artifact?”, American Journal of Political Science, vol. 36, núm. 1, 1992, p. 175.

${ }^{6}$ Carlos J. Vilalta, "The Local Context and the Spatial Diffusion of Multiparty Competition in Urban Mexico, 1994-2000”, Political Geography, vol. 23, núm. 4, 2004. 
etc.) puede tener opiniones, percepciones e intereses únicos, e inclusive opuestos a las tendencias nacionales, sea cual sea la composición del lugar. ${ }^{7}$ Estas opiniones localmente mayoritarias pueden a su vez influir en las opiniones individuales, y viceversa. Es decir, se acepta la posibilidad causal que puede tener el "lugar" en el comportamiento; gente aparentemente similar puede tener una opinión diferente dependiendo de dónde vive. ${ }^{8}$

Esta perspectiva del "efecto contextual" se fundamenta en la teoría de la estructuración de Giddens. ${ }^{9}$ Esta teoría postula que tanto la agencia (o capacidades individuales) como la estructura (o normas y recursos) se afectan mutuamente. Mientras que la agencia es influenciada por las "tradiciones, instituciones, códigos morales y maneras establecidas de hacer las cosas", ${ }^{10}$ a su vez los individuos transforman las estructuras al ignorarlas, sustituirlas o reproduciéndolas de manera diferente a través del tiempo y el espacio. Giddens ${ }^{11}$ sostiene que los individuos se hallan envueltos en procesos constantes de cambio de comportamiento y que estas transformaciones incluyen, por supuesto, cambios en la opinión sobre un problema socialmente compartido.

Una implicación geográfica de la teoría de la estructuración es que los procesos sociales no son geográficamente aleatorios. Los

${ }^{7}$ John A. Agnew, Place and Politics: The Geographical Mediation of State and Society, Londres, Allen and Unwin, 1987; John A. Agnew, "The Territorial Trap: The Geographical Assumptions of International Relations Theory", Review of International Political Economy, vol. 1, 1994; Michael E. Shin y John A. Agnew, "The Geography of Party Replacement in Italy, 1987-1996”, Political Geography, vol. 21, 2002.

${ }^{8}$ Carlos J. Vilalta, "Perspectivas geográficas en la sociología urbana: La difusión espacial de las preferencias electorales y la importancia del contexto local”, Estudios Demográficos y Urbanos, vol. 18, núm. 3, 2003.

${ }^{9}$ Anthony Giddens, “Agency, Institution, and Time-Space Analysis”, en Karin Knorr-Cetina y Aaron. V. Cicourel (eds.), Advances in Social Theory and Methodology: Toward An Integration of Micro- and Macro-Sociologies, Boston, Routledge \& Kegan Paul, 1981; Anthony Giddens, The Constitution of Society, Berkeley, University of California Press, 1984; Anthony Giddens, The Consequences of Modernity, Stanford, Standford University Press, 1990.

${ }^{10}$ Anthony Giddens y Christopher Pierson, Conversations with Anthony Giddens: Making Sense of Modernity, Cambridge, Polity Press, 1998, p. 77.

${ }^{11}$ Giddens, The Consequences of Modernity. 
procesos sociales suceden en un contexto local que posee causas y efectos (agregados) de comportamiento, percepción y opinión. Es decir, ni la percepción, ni la opinión, ni el comportamiento son creados en un vacío social. Los procesos sociales crean un contexto, el cual a su vez crea procesos sociales. La vida social (o sistema social) no es una simple suma de micro-acciones individuales, sino el resultado de una relación continua entre estructura y agencia. ${ }^{12} \mathrm{Al}$ igual que la estructura afecta el comportamiento de los agentes, los agentes a su vez transforman las estructuras. Esto es, en términos simples, lo que propone la teoría de la estructuración de Giddens. ${ }^{13}$

El contexto local se ha definido como el conjunto de "redes sociales, fuentes de información y de referencia de grupos asentados en ciertos lugares". ${ }^{14}$ Esto es lo que Huckfeldt y Sprague ${ }^{15}$ llamaron hace muchos años behavioral contagion. Este es el fundamento propio de la geografía humana; consiste en el supuesto de que la población residente en un lugar o región tiende a ser más similar entre ella en sus comportamientos, percepciones y opiniones, a consecuencia de la suma de interacciones de las instituciones locales (ej. familia, amistades, escuela, trabajo, etc.), que, precisamente sumadas, crean el contexto local de los individuos. Este fundamento obviamente coincide con la primera ley de la geografía de Tobler $^{16}$ en la idea de que todas se relacionan, pero las cosas cercanas son más similares entre ellas que las cosas lejanas. Y esta ley es obviamente aplicable a todas las ramas de la geografía, incluida la geografía política cuando hablamos de comportamientos y opiniones al respecto de la "polis".

12 Giddens, The Constitution of Society.

${ }^{13}$ Loc. cit.

${ }^{14}$ Richard Sauerzopf y Todd Swanstrom, "The Urban Electorate in Presidential. Elections, 1920-1996”, Urban Affairs Review, vol. 35, 1999, p. 87.

${ }^{15}$ Robert Huckfeldt y John Sprague, "Political Parties and Electoral Mobilization: Political Structure, Social Structure, and the Party Canvass", American Political Science Review, marzo de 1992.

16 Waldo R. Tobler, "A Computer Movie Simulation Urban Growth in the Detroit Region”, Economic Geography, vol. 46, núm. 2, 1970. 


\section{2 ¿Qué es el lugar y qué es el espacio?}

De forma más general, el lugar es la distinción entre aquí y allá. ${ }^{17}$ $\mathrm{Y}$ es tan evidente a nuestra vista y sensación, que normalmente lo perdemos de vista. Pero los lugares son distintivos en los tres elementos que los constituyen: ubicación, forma material y significado o valor. ${ }^{18}$

La ubicación es la posición en el espacio. La ubicación geográfica siempre está dada por latitud y la longitud en un plano. Un lugar siempre tiene una ubicación dada por sus coordenadas. A su vez, una forma material es cualquier cosa con una representación física. Así, un lugar tiene una representación física en, por ejemplo, una calle, un parque, un barrio, una ciudad, etc. Sin embargo, los lugares son más que una ubicación con una forma material; los lugares tienen un significado o existencia emocional. ${ }^{19}$ En los lugares, la gente crea sus propias creencias, normas y vive sus emociones también. ${ }^{20}$ Lo anterior no sólo es consecuencia de eventos presentes, sino también de un pasado que conforma a las llamadas "historias locales", que son, básicamente, acumulaciones de eventos pasados que dan sentido en muchas ocasiones a los eventos presentes. Estos eventos, a lo largo del tiempo, se reflejan materialmente en el llamado espacio construido, con que muestran estilos e ideologías arquitectónicas de épocas diferentes, dando a los lugares elementos estéticos distintivos provenientes de patrones de socialización observables.

Los lugares son redes sociales, ${ }^{21}$ las cuales evidentemente son geográficamente variables. Esto es importante de entender para efectos de un análisis no sólo geográfico, sino sociológico también,

17 Thomas F. Gieryn, "A Space for Place in Sociology”, Annual Review of Sociology, vol. 26, 2000.

${ }^{18}$ Loc. cit.

${ }^{19}$ Carlos J. Vilalta, "How Exactly Does Place Matter in Crime Analysis? Place, Space and Spatial Heterogeneity", Journal of Criminal Justice Education, vol. 24, núm. 3, 2013.

${ }^{20}$ Agnew, Place and Politics.

${ }^{21}$ Doreen B. Massey, Space, Place, and Gender, Minneapolis, University of Minnesota Press, 1994. 
puesto que las relaciones sociales estructuran nuestras percepciones y opiniones individuales. En síntesis: el lugar es una variable independiente. Es circunstancial y culturalmente dinámica. No es algo concreto y culturalmente estático. Los lugares son también categorías de análisis sociológico o político, porque nos ayudan a organizar, y por ende a entender, nuestra vida emocional.

Ahora bien, un lugar evidentemente requiere de un espacio en el cual existir. Nótese que un espacio es algo, cualquier cosa, con una superficie y volumen. Su etimología es bien conocida e interesante. En latín clásico, spatium es sinónimo de ambitus (o ámbito), en tanto que ambos sirven para expresar una extensión o una circunferencia de algo. ${ }^{22}$ Así, un espacio siempre tiene atributos geométricos (ej. área, volumen), pero no tiene un significado cultural ni atributos de valor. Donde los lugares son objetos de estudio, los espacios son contenedores de ese objeto de estudio; los lugares son unidades de análisis y los espacios son unidades de información. ${ }^{23}$ Por ejemplo, un área geo-estadística básica (AGEB), que es una subdivisión del territorio nacional para efectos de muestreo y recopilación de datos censales por parte del Instituto Nacional de Estadística y Geografía (INEGI), es un caso de espacio o unidad de información, pero no podemos decir que es un lugar en cuanto a que no tiene un significado para los residentes en esta subdivisión; nadie se ubica en un AGEB, pero todos sí nos podemos ubicar en una colonia o un municipio.

Finalmente, y relacionado con la sección previa, se debe aclarar por qué el lugar sirve como variable independiente. Por los elementos previamente mencionados, los lugares organizan la forma en que nos comportamos. ${ }^{24}$ El lugar es así un concepto organizador clave, o unidad de análisis como se dijo hace un momento, para la investigación social. Es decir, los lugares son unidades de

${ }^{22}$ Stuart Elden, "Land, Terrain, Territory", Progress in Human Geography, vol. 34,2010 .

${ }^{23}$ Vilalta, "How Exactly Does Place Matter in Crime Analysis?".

24 Pred, A., "Context and Bodies in Flux: Some Comments on Space and Time in the Writings of Anthony Giddens", en J. Clark, C. Modgil y S. Modgil (eds.), Anthony Giddens: Consensus and Controversy, Londres, The Falmer Press, 1990, pp. 117-129. 
análisis cuando se entienden como "patrones de relaciones", es decir, como estructuras. Y aquí aparece de nuevo la teoría de la estructuración para justificar la importancia de la geografía o el contexto local en el estudio de la opinión: el lugar o contexto local es la interacción entre el individuo y su medio "con la naturaleza del medio ambiente siendo construido a través del tiempo por los mismos actores". ${ }^{25}$ Desde esta perspectiva, ninguna explicación puede ser solamente estructural o agencial.

\section{Caso de ESTUdio: la AMENAZA del naRcotráfico Y EL CRIMEN ORGANIZADO EN MÉXICO}

El narcotráfico y el crimen organizado constituyen un problema muy grave. Para entender la gravedad de este problema, repasemos brevemente información proveniente de cuatro diferentes fuentes relacionadas: actividad policial, actividad judicial, el discurso oficial y la opinión pública. Además de lo anterior, en esta sección se revisan algunos estudios previos relacionados con la amenaza del narcotráfico y el crimen organizado.

Empezando con la actividad policial, en un día cualquiera de 2013 se iniciaban cuatro averiguaciones previas por algún delito relacionado con delincuencia organizada y 24 por algún delito contra la salud relacionado con el narcotráfico. ${ }^{26}$ Esta es una cifra considerable por todas sus implicaciones; representa una política federal criminal extensiva que usa cantidades de recursos considerables.

Pero esto no debe sorprendernos como un problema nuevo en estos mismos términos criminológicos. Al menos desde 2001 (año desde el cual contamos con series de datos para estos delitos), éste ya era un problema de seguridad pública serio. Básicamente, en este periodo de doce años, una de cada tres averiguaciones previas por la comisión de algún delito federal se relacionaba con algún

${ }^{25}$ Flint, op. cit.

${ }^{26}$ Estos datos consideran delitos contemplados en el Código Penal Federal y en contra de la Ley General de Salud relacionados con el narcotráfico en alguna de las siguientes modalidades: comercio, posesión, producción, suministro, tráfico, transporte u otro relacionado. 
hecho delictivo relacionado con el narcotráfico. En números absolutos estamos hablando de más de medio millón de averiguaciones previas iniciadas desde entonces, sólo por esos delitos. Y es la modalidad de posesión la que ha llevado el grueso de las averiguaciones $(74.8 \%)$. Estas cifras muestran, además, las notables diferencias de enfoque de política criminal en los últimos periodos presidenciales.

\section{Cuadro 1}

Delitos federales, delincuencia organizada y delitos contra la salud: averiguaciones previas iniciadas por año, 2001 a 2013

\begin{tabular}{cccccc}
\hline & $\begin{array}{c}\text { Delitos } \\
\text { Federales }\end{array}$ & $\begin{array}{c}\text { Delincuencia } \\
\text { Organizada (Do) }\end{array}$ & $\begin{array}{c}\text { Contra la Salud } \\
(\text { DCS })\end{array}$ & $\begin{array}{c}\text { Por Posesión } \\
(\text { DCS })\end{array}$ & $\begin{array}{c}\text { Posesión en } \\
\text { los DCS (\%) }\end{array}$ \\
\hline 2001 & 74113 & 217 & 23232 & 18350 & $79.0 \%$ \\
2002 & 73803 & 447 & 23588 & 18295 & $77.6 \%$ \\
2003 & 81230 & 669 & 28645 & 21595 & $75.4 \%$ \\
2004 & 81539 & 881 & 28715 & 19795 & $68.9 \%$ \\
2005 & 89530 & 611 & 38903 & 29131 & $74.9 \%$ \\
2006 & 109629 & 403 & 58066 & 49255 & $84.8 \%$ \\
2007 & 137289 & 1217 & 81491 & 71511 & $87.8 \%$ \\
2008 & 136091 & 1165 & 73222 & 47535 & $64.9 \%$ \\
2009 & 131582 & 1505 & 63404 & 43326 & $68.3 \%$ \\
2010 & 132227 & 1950 & 55122 & 39180 & $71.1 \%$ \\
2011 & 133045 & 2505 & 42747 & 31665 & $74.1 \%$ \\
2012 & 125328 & 2270 & 27870 & 19643 & $70.5 \%$ \\
2013 & 98189 & 1302 & 8836 & 5009 & $56.7 \%$ \\
General & 1403595 & 15142 & 553841 & 414290 & $74.8 \%$ \\
\hline
\end{tabular}

Fuente: Elaboración propia con base en datos del Secretariado Ejecutivo del Sistema Nacional de Seguridad Pública (SESNsP) y en información de la Procuraduría General de la República (PGR).

Estos incrementos en la actividad policial se reflejan naturalmente en incrementos de la actividad judicial. Entre 1997 y 2012, poco más de 600000 individuos fueron procesados por algún delito relacionado con narcóticos, los cuales constituyen la mayoría de los delitos contra la salud. También se observa que la proporción 
de procesados en el ámbito federal por estos delitos fue progresivamente aumentando, para eventualmente constituir $42 \%$ del total de los procesados en éste ámbito de la justicia penal federal. Es, sin duda, el tipo de delito al que más recursos y atención se le destinan por parte del poder federal cada año.

Cuadro 2

Delitos federales en materia de narcóticos: procesados por año, 1997 a 2012

\begin{tabular}{cccc}
\hline & Total en el fuero federal & En materia de narcóticos & En materia de narcóticos (\%) \\
\hline 1997 & 34740 & 12266 & $35.3 \%$ \\
1998 & 34101 & 12098 & $35.5 \%$ \\
1999 & 33981 & 12754 & $37.5 \%$ \\
2000 & 32260 & 12077 & $37.4 \%$ \\
2001 & 31378 & 11488 & $36.6 \%$ \\
2002 & 33599 & 12433 & $37.0 \%$ \\
2003 & 35575 & 14001 & $39.4 \%$ \\
2004 & 35010 & 14516 & $41.5 \%$ \\
2005 & 36892 & 16828 & $45.6 \%$ \\
2006 & 35492 & 17431 & $49.1 \%$ \\
2007 & 36941 & 17889 & $48.4 \%$ \\
2008 & 43602 & 18529 & $42.5 \%$ \\
2009 & 48279 & 19652 & $40.7 \%$ \\
2010 & 52514 & 26155 & $49.8 \%$ \\
2011 & 56545 & 25487 & $45.1 \%$ \\
2012 & 50383 & 21504 & $42.7 \%$ \\
General & 631292 & 265108 & $42.0 \%$ \\
\hline
\end{tabular}

Fuente: Elaboración propia con base en datos del Sistema de Estadísticas Judiciales (INEGI).

Es de esperar que el discurso oficial se relacione, en los hechos, con la actividad policial y judicial previamente descritos. El discurso, sobre todo el de los últimos años, no solamente ha reconocido la magnitud del problema, sino que lo ha posicionado como un tema prioritario en la agenda nacional. $\mathrm{Al}$ inicio del sexenio 20062012, el presidente Felipe Calderón lanzó una nueva estrategia en 
el combate a la inseguridad, y en específico contra el crimen organizado, la cual fue siempre presentada en multitud de discursos y comunicados durante esos seis años. La delincuencia organizada fue el tema de mayor importancia en la agenda de ese sexenio; siempre fue el tema prioritario desde los primeros días de la administración. El 30 de noviembre de 2006 se hizo la declaración del combate, durante la presentación del gabinete de seguridad, y el día siguiente el presidente declaró formalmente la estrategia a seguir. La siguiente transcripción de un comunicado hecho público a mediados del sexenio resume perfectamente bien el discurso oficial de la estrategia, en sus motivos e intenciones: ${ }^{27}$

Uno de los desafíos más importantes que enfrenta México es el de la inseguridad $[\ldots]$ se trata de una problemática que se ha venido exacerbando con el tiempo [...] Para responder a este desafío, el gobierno federal ha decidido enfrentar con determinación al crimen organizado desde el inicio de esta Administración. El objetivo ha sido recuperar la seguridad de las familias mexicanas; refrendar a México como un país de leyes y de instituciones; y garantizar una convivencia ordenada y tranquila en todo el país [...]

Hay, por cierto, una ordenación lógica y muy clara de las causas en el discurso oficial que explican bien la amenaza que enfrentamos, y que sistematizo a continuación por la utilidad que tiene para comprender nuestro estudio de caso y porque ayuda a explicar que el discurso oficial siempre fue claro y siempre fue dirigido a impactar en la opinión pública. El propósito principal fue priorizar el tema y legitimar la estrategia. Lo primero fue ciertamente logrado, pero lo segundo siempre se debatió.

En síntesis, al respecto de la amenaza del crimen organizado, el discurso oficial del sexenio pasado siempre contuvo todos los elementos del discurso político, es decir, una justificación, una suma de circunstancias y de hechos, además de una fuerte carga simbólica. ${ }^{28}$

${ }^{27}$ Véase http://calderon.presidencia.gob.mx/2010/06/la-lucha-por-la-seguridad-publica/

${ }^{28}$ Por ejemplo, cuando el presidente Calderón apareció con partes de uniforme 


\section{GRÁFICA 1}

El discurso oficial de las causas de la amenaza del narcotráfico y el crimen organizado desde el discurso oficial, 2006-2012

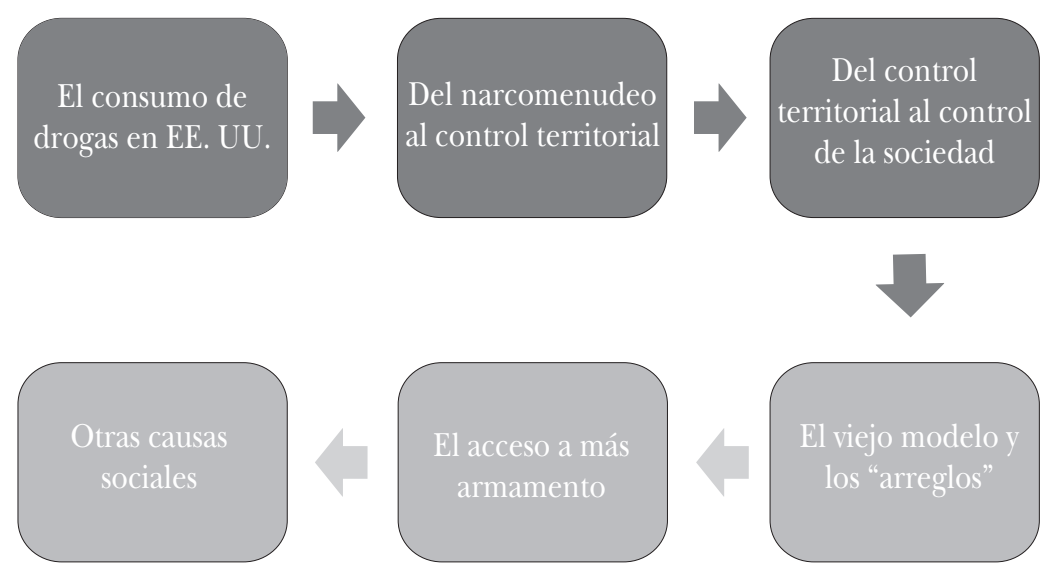

Fuente: Capitulado de las causas contenidas en el comunicado presidencial del 13 de Junio del año 2010.

Es imposible que la opinión pública no fuera impactada. Dados los hechos y la retórica de Estado, era de esperarse el efecto. Pero, de nuevo, la opinión o percepción ciudadana al respecto no es nueva, ni tampoco lo era el sexenio pasado; lo que sí sucedió es que tal percepción fue agravándose progresivamente desde hace dos décadas, para hacerse más grave estos últimos seis o siete años.

Por ejemplo, investigando históricamente al respecto, encontramos registro de que en 1995 , solamente $11.3 \%$ de la población residente en la zona metropolitana de la ciudad de México consideraba que el país se hallaba amenazado por el narcotráfico o la delincuencia en general. ${ }^{29}$ Unos años después, en

militar en un evento realizado en reconocimiento a policías y militares en Apatzingán el 3 de enero de 2007, en donde, no sobra decir, siete años después y mientras se escriben estas líneas, hay fuertes enfrentamientos entre cárteles, grupos de autodefensa y cuerpos de seguridad del Estado. Es un escenario que precisamente muestra la gravedad de la amenaza del crimen organizado para el país.

${ }^{29}$ En concreto, solamente el $1.4 \%$ opinaba que el narcotráfico era una amenaza, mientras que la delincuencia (en general) era el 9.9\%. En este caso suma- 
2001, ya un 52\% de los residentes adultos del país era de la opinión de que el narcotráfico era "un problema grave y se complicará en el futuro". ${ }^{30}$ Inclusive, la misma encuesta de México, las Américas y el Mundo que se analiza en el presente estudio ha registrado esta preocupación desde el año 2004. En todos los levantamientos bienales que se han hecho desde entonces, la proporción de la población adulta que ha considerado al narcotráfico y al crimen organizado como una "amenaza grave" siempre ha estado alrededor o por encima de $80 \%$ (hasta donde lo permite inferir el margen de error y la metodología propia de cada levantamiento).$^{31}$

Resumiendo, es de esperarse que los hechos y el discurso oficial posicionaran un tema en la agenda pública y que, mutua y circularmente, impactara en la opinión pública. Esto se debe a que el narcotráfico y el crimen organizado representan, en los hechos, los mensajes de gobierno y la opinión pública, una amenaza verdadera para la paz y la estabilidad del país. Pero veremos que la opinión pública de tal amenaza no es la misma a lo largo del país. La opinión, si bien es grave, no es la misma para todos y depende de la región en donde se viva. A continuación se desarrollan la hipótesis y la metodología que se siguen en este estudio para poner a prueba la anterior aseveración.

Entre los estudios que han analizado estos datos y otros, y han hablado de las variaciones regionales al respecto de la amenaza del crimen organizado y el narcotráfico, destacan los trabajos de

mos las dos proporciones. Fuente: Encuesta de Seguridad Pública y Justicia. Levantada el 15 de julio de 1995 por la Oficina de la Presidencia de la República Mexicana. Base de datos disponible en: http://biiacs-dspace.cide.edu/handle/10 089/3498

${ }^{30}$ Fuente: Encuesta de Evaluación y percepción de la inseguridad en el país. Levantada el 30 de marzo de 2001 por la Oficina de la Presidencia de la República Mexicana. Base de datos disponible en: http:/ / biiacs-dspace.cide.edu/handle/10 $089 / 15997$

${ }^{31}$ Léanse estos datos con suficiente cautela; las entidades federativas en donde fueron aplicadas las encuestas no son las mismas para todos los años. Esto se explica con detalle en la sección de hipótesis y metodología del presente estudio. 
Magaloni y colaboradores ${ }^{32}$ y Vilalta ${ }^{33}$ por su enfoque en la percepción del problema. Si bien no se encontró un estudio similar que se enfocara directamente en la percepción de la amenaza del

\section{GRÁFICA 2}

Proporción de encuestados que consideran al narcotráfico y el crimen organizado como una amenaza grave para el país, 2004 a 2012

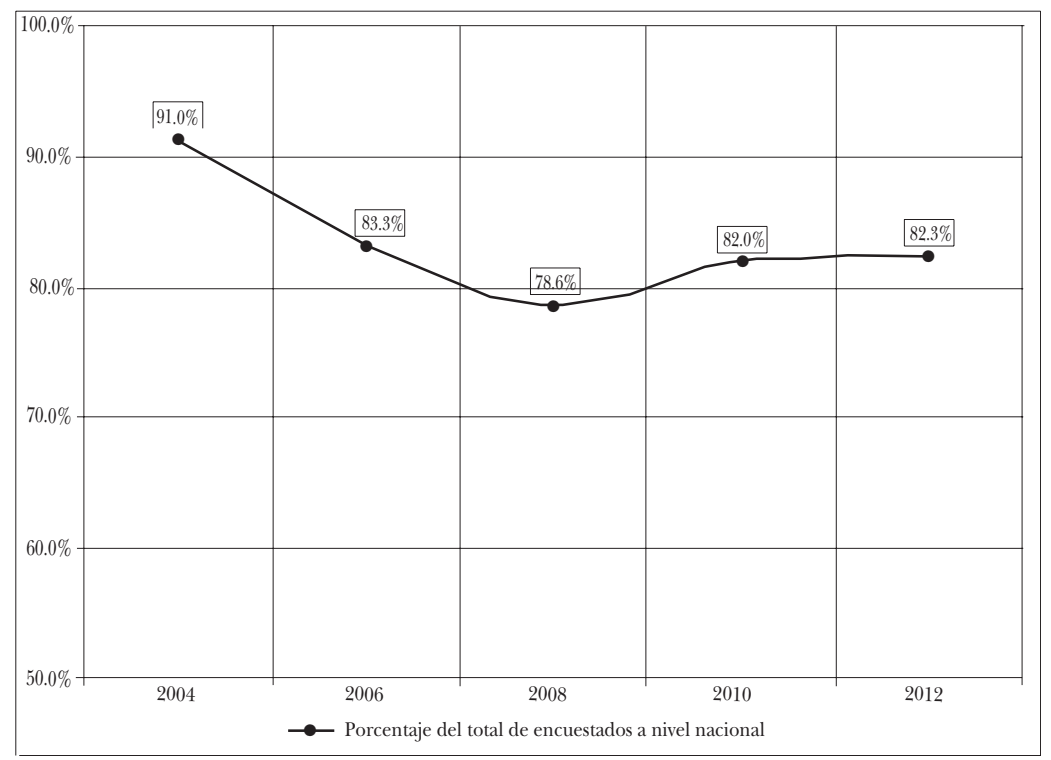

Fuente: cálculos propios con base en las encuestas de México, Las Américas y el Mundo, 2004, 2006, 2008, 2010 y 2012.

${ }^{32}$ B. Magaloni, A. Díaz-Cayeros y V. Romero, "La raíz del miedo: ¿Por qué es la percepción de riesgo mucho más grande que las tasas de victimización?”, en José Antonio Aguilar (coord.), Las bases sociales del crimen organizado, México, Secretaría de Seguridad Pública (ssP) y Centro de Investigación en Estudios en Seguridad (CIES), 2012, capítulo 4.

${ }^{33}$ Vilalta, "Does the Mexican War on Organized Crime Mediate the Impact of Fear of Crime on Daily Routines?", Crime E Delinquency, 2014. DoI: 10.1177/001 1128714541208 . 
crimen organizado y el narcotráfico, sí se encontraron otros estudios relacionados como los de Magaloni y colaboradores, quienes reportan que el contexto local importa para determinar el nivel de miedo al crimen, pero de una forma sorpresiva, es decir, que los individuos residentes en zonas menos afectadas por los homicidios relacionados con el combate al crimen organizado son menos propensos a reportar tener miedo al delito. A la inversa, el estudio de Vilalta encuentra un resultado opuesto en cuanto a que los residentes en las zonas más afectadas por muertes también asociadas al combate al crimen organizado sí reportan tener mayores niveles de inseguridad y de miedo al crimen frente a los residentes en zonas menos afectadas por este problema, aun controlando por otros correlativos clásicos en la literatura de la percepción y miedo al delito. ${ }^{34}$ Es posible que la diferencia en los hallazgos tenga que ver con que utilizan fuentes de información diferentes.

\section{Hipótesis y METODOLOGÍA}

Este estudio se centra en analizar la opinión que la población adulta del país tiene al respecto del narcotráfico y crimen organizado como una amenaza para el país en su conjunto. Además de saber cuál es tal opinión y su variación en el tiempo, interesa saber los motivos de las variaciones regionales que más adelante mostramos. Por lo que, dado el debate teórico previamente presentado, la hipótesis de investigación en este estudio, presentada a continuación en términos de hipótesis nula, sería la siguiente:

Ho: el lugar o contexto local no tiene una relación con la conformación de la opinión al respecto de la amenaza del narcotráfico y el crimen organizado.

De esta forma, la prueba de la hipótesis permite dar una respuesta directa a la siguiente pregunta derivada del debate: ¿ison las

${ }^{34}$ Vilalta, "Does the Mexican War on Organized Crime Mediate the Impact of Fear of Crime on Daily Routines?". 
variaciones regionales de opinión aleatorias?, ¿o con qué se asocian? ¿Con la composición poblacional de cada región o con el contexto local o con ambas? Es decir, ¿somos similares en nuestra opinión una vez considerada nuestra composición demográfica y socioeconómica, o bien opinamos de forma diferente independientemente de tal composición y según donde residamos?

Para probar la hipótesis anterior y dar respuesta a estas preguntas, se analizó la encuesta México, las Américas y El Mundo de los años 2008, 2010 y 2012. El motivo de utilizar estos años específicos son los cambios en el número de estados que conforman las regiones dominio de la encuesta por vuelta de la misma. Los levantamientos de 2004 y 2006 se conforman por 23 estados divididos en 3 regiones; para los siguientes años, las tres regiones consideraron entre 26 y 32 estados para realizar sus estimaciones (véase el cuadro 3).

\section{Cuadro 3}

Encuesta de México, las Américas y el Mundo: estados contenidos por región por vuelta de la encuesta, 2004 a 2012

\begin{tabular}{ccccc}
\hline & Norte-Frontera & Sur-Sureste & Resto del país & Total \\
\hline 2004 & 6 & 5 & 12 & 23 \\
2006 & 6 & 5 & 12 & 23 \\
2008 & 6 & 7 & 19 & 26 \\
2010 & 6 & 7 & 18 & 31 \\
2012 & 6 & 7 & 19 & 32 \\
\hline
\end{tabular}

Fuente: elaboración propia con base en información de las encuestas de México, las Américas y el Mundo de 2008, 2010 y 2012.

Todo análisis geográfico requiere, idealmente, que las unidades de análisis territorial sean las mismas a través del tiempo. En esta misma línea metodológica, para hacer efectivamente comparables las regiones en 2008, 2010 y 2012, se tuvo que sustraer a los estados de Baja California Sur y Tamaulipas del análisis estadístico. El motivo es que el estado de Tamaulipas no fue considerado el año de 2010 y que el estado de Baja California Sur fue considerado dentro 
de la región "Resto del país" en 2008, pero dentro de la región "Norte" en 2010 y 2012. En este aspecto, es obviamente inadecuado "mover" estados entre regiones, entre años, de la encuesta, siendo que las ponderaciones regionales de la encuesta están precisamente diseñadas por los estados constituyentes de cada región. Estadísticamente, no es correcto asignar un peso o ponderación de una región en su conjunto a poblaciones de un estado en otra región con otra ponderación original.

\section{GRÁFICO 3}

Mapa de los estratos regionales

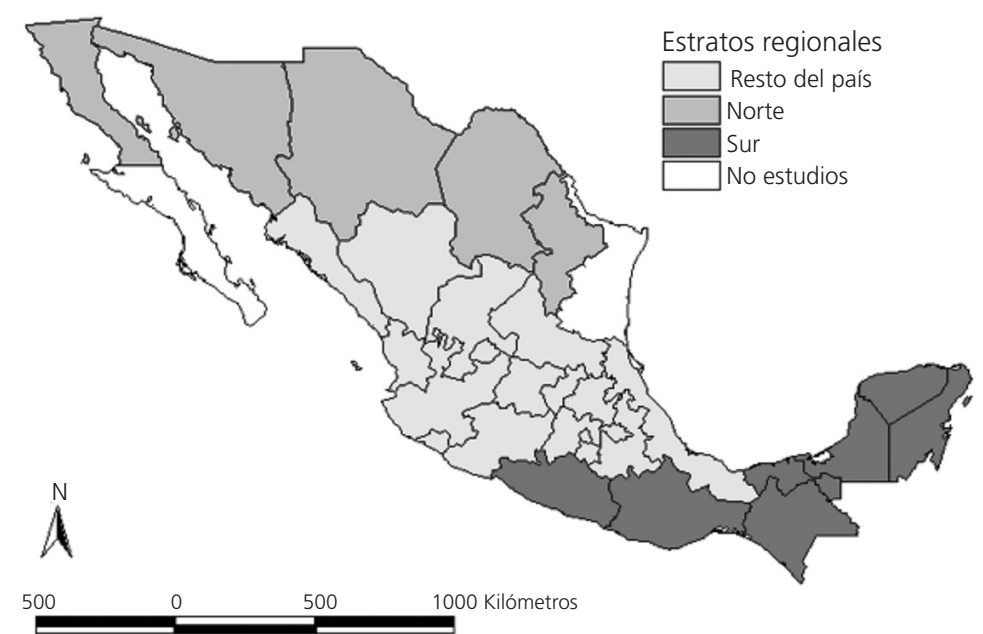

Fuente: elaboración propia con base en información de las encuestas de México, las Américas y el Mundo de 2008, 2010 y 2012.

La variable dependiente es la opinión al respecto del narcotráfico y el crimen organizado como una amenaza para el país. La formulación de la pregunta respectiva en el cuestionario, en este caso, no varió entre años (véase el cuadro 4), lo que proporcionó el beneficio de la comparabilidad, una vez resueltas las limitaciones en las regionalizaciones previamente mencionadas. 


\section{Cuadro 4}

Variable dependiente: formulación de la pregunta y las respuestas en la encuesta, 2008, 2010 y 2012

\begin{tabular}{ccc}
\hline Año & \multicolumn{1}{c}{ Pregunta } & Respuesta \\
\hline 2008 & Le voy a leer una lista de asuntos que & (1) Amenaza grave \\
pueden o no afectar a los intereses más & (2) Amenaza importante pero no \\
importantes de México en los próximos & muy grave \\
10 años. Dígame si, para cada uno, us- & (3) Amenaza poco importante \\
ted percibe... (el narcotráfico y el cri- & (4) No es una amenaza (espontánea) \\
men organizado)... como una amena- & (8) No sabe \\
za grave, amenaza importante pero no & (9) No contestó \\
grave o una amenaza poco importante & \\
para México & \\
Le voy a leer una lista de asuntos que & (1) Amenaza grave \\
pueden o no afectar a los intereses más & (2) Amenaza importante pero no \\
importantes de México en los próximos & muy grave \\
10 años. Dígame si usted percibe... (el & (3) Amenaza poco importante \\
narcotráfico y el crimen organizado)... & (4) No es una amenaza (espontánea) \\
como una amenaza grave, amenaza im- & (8) No sabe \\
portante pero no grave o una amenaza & (9) No contestó \\
poco importante para México & \\
& & \\
Le voy a leer una lista de asuntos que & (1) Amenaza grave \\
pueden o no afectar a los intereses más & (2) Amenaza importante pero no \\
importantes de México en los próximos & muy grave \\
diez años. Dígame si usted percibe... & (3) Amenaza poco importante \\
(el narcotráfico y el crimen organiza- & (4) No es una amenaza (espontánea) \\
do)... como una amenaza grave, ame- & (8) No sabe \\
naza importante pero no grave o una & (9) No contestó \\
amenaza poco importante para México & \\
\hline
\end{tabular}

Fuente: elaboración propia con base en información de las encuestas de $M e^{-}$ xico, las Américas y el Mundo de 2008, 2010 y 2012.

Para explicar cuál fue la variable independiente y cuáles las variables de control, se explica a continuación el procedimiento que se siguió para la prueba de hipótesis, lo cual facilita la distinción entre ellas. La prueba se realizó en varios pasos. Primero se buscó evidencia de diferencias regionales por medio de pruebas inferenciales de diferencias para cada año analizado. Las regiones 
son las mismas de los estratos de la encuesta para cada año y que se muestran en el mapa anterior (norte, sur y resto del país). Se utilizó la prueba $\mathrm{F}$ para medias aritméticas para comparar a las regiones y al cambio dentro de cada región. ${ }^{35}$ Una vez confirmadas las diferencias estadísticamente significativas entre las regiones y en las regiones a lo largo del tiempo, se procedió a realizar una prueba de un modelo correlacional multivariado por medio de una regresión logística ordinal siendo que la variable dependiente tiene (véase el cuadro 4) una escala ordinal. Además de los diagnósticos tradicionales de significancia del modelo y de capacidad predictiva, se agregó una prueba de significancia de líneas paralelas para cumplir con el criterio de que los coeficientes de las pendientes sean los mismos (o paralelos) para cada nivel de la variable dependiente.

En el modelo correlacional, la variable independiente fueron las regiones (o estratos de la muestra) que corresponden con el lugar de residencia de los adultos encuestados, y las variables de control fueron una variedad de variables composicionales contenidas en la misma encuesta. Estas variables son el género, el grupo de edad, el grado de escolaridad, el nivel de ingreso, la posesión de teléfono fijo o celular y el uso de Internet. Las últimas tres variables de control dirigidas a medir el uso y acceso de medios para la telecomunicación son particularmente útiles para someter a la hipótesis nula una prueba más rigurosa, dado el argumento de la teoría de la Nacionalización de que el espacio o distancia entre los individuos no es importante para predecir el comportamiento o la opinión política dada la modernización con la correspondiente homogeneización de los estilos de vida y el acceso a mayores niveles de información masiva en las sociedades modernas frente a las sociedades tradicionales.

Se corrió una regresión para cada año. Es decir, el modelo se probó para los años 2008, 2010 y 2012. Esto se hizo para efectos de someter la hipótesis a una mayor condición de evidencia disponible, y ofrecer así una mayor certidumbre, en la medida de lo

${ }^{35}$ Se utilizó esta prueba paramétrica pese a que la variable dependiente tiene una escala originalmente ordinal. Esta prueba se utilizó a petición de dos árbitros de mostrar los intervalos de confianza alrededor de la media de esta variable. 
posible, de la plausibilidad de la importancia del lugar o contexto local. Se considera que la prueba repetida de la misma hipótesis en el tiempo da una mayor solidez a las conclusiones del estudio. Además del análisis multivariado se hizo uso de estadísticos descriptivos y mapas para una mejor exposición del objeto de estudio. ${ }^{36}$ Los resultados del análisis se presentan a continuación.

\section{Resultados}

\subsection{Tendencias nacionales y variaciones regionales}

Entre 2008 y 2012, la percepción de la gravedad del problema del narcotráfico y el crimen organizado como una amenaza grave para el país se hizo más común o más compartida entre la población adulta del país. Entre 2008 y 2010, cada vez fueron más los individuos que calificaron este problema como una amenaza nacional. Notablemente, la opinión entre 2010 y 2012 no cambió significativamente, pese a que para el último año ya se contaba con más información y elementos para juzgar si la guerra contra el crimen organizado había tenido algún éxito en revertir tal amenaza.

Nótese con todo que la opinión agregada nacionalmente no es la misma que la opinión de todas las regiones. La percepción del grado de amenaza del narcotráfico y el crimen organizado contra el país varía significativamente entre las regiones representadas en la encuesta de México, las Américas y el Mundo. Las diferencias regionales se detectaron para los años 2008 y 2010, pero no para 2012. Igualmente, se detectó que la opinión se modificó a través del tiempo en la región sur y para el agregado nacional, mientras que para los habitantes de la región norte y del resto del país, la percepción de la gravedad del problema del narcotráfico y el crimen organizado como una amenaza grave para el país se mantuvo estadísticamente sin cambio.

${ }^{36}$ Además, el anexo contiene los resultados una prueba geográfica accesoria sobre el desplazamiento de la opinión sobre el territorio. 
Empezando por los residentes en la región sur del país, podemos ver que si bien en 2008 la opinión era la menos negativa al respecto en comparación con las demás regiones, para el año 2012 esta población poseía una opinión similar de una alta gravedad o de mayor amenaza del narcotráfico y el crimen organizado. Póngase estas cifras en contexto. Nótese que la opinión, todos los años y en todas las regiones, ha sido muy negativa. En una escala de 1 a 4 en donde 1 representa una opinión de "amenaza grave para el país" y 4 "no es una amenaza”, de forma sistemática y para todas las regiones, el promedio nunca ha sido menor de 1.32. En este sentido, son los habitantes de la región sur del país los que más han variado en su opinión a lo largo de estos cuatro años y en dirección a percibir una mayor amenaza.

\section{Cuadro 5}

El narcotráfico y el crimen organizado como una amenaza para el país: medias aritméticas, 2008, 2010 y 2012

\begin{tabular}{lcccc}
\hline & 2008 & 2010 & 2012 & $\begin{array}{c}\text { Prueba entre años al } \\
\text { interior de la región }\end{array}$ \\
\hline Norte & 1.24 & 1.26 & 1.21 & 0.736 \\
Sur & $(1.20-1.28)$ & $(1.21-1.31)$ & $(1.15-1.26)$ & 1.16 \\
Resto del país & 1.32 & 1.15 & $1.456 * * *$ \\
& $(1.27-1.38)$ & $(1.11-1.19)$ & $(1.09-1.21)$ \\
Nacional & $(1.21-1.27)$ & $(1.17-1.22)$ & $(1.18-1.26)$ & 2.258 \\
& 1.26 & 1.20 & 1.20 & $9.918 * * *$ \\
Prueba entre & $(1.24-1.28)$ & $(1.18-1.22)$ & $(1.17-1.23)$ \\
regiones cada año & $4.008 * *$ & $4.348^{* *}$ & 1.305 & - \\
\hline \multicolumn{5}{c}{$* * * \mathrm{p}<0.01, * * \mathrm{p}<0.05, * \mathrm{p}<0.10$} \\
\end{tabular}

Nota: se muestran las medias aritméticas para todas las opciones posibles de respuesta donde 1 significa "Amenaza grave" y 4 significa "No es una amenaza". Es decir, un valor bajo o cercano a 1 significa ser una amenaza grave. No se consideraron las NR o NS. La prueba entre años al interior de cada región es una prueba F o anova de diferencias de medias aritméticas. En paréntesis los intervalos de confianza de la media al $95 \%$. 
De forma similar, los habitantes en el norte del país también han opinado gravemente al respecto; su opinión no varió significativamente durante 2008 y 2012. Únicamente en 2010, los residentes de esta región tuvieron una opinión de menor gravedad de la amenaza del narcotráfico y el crimen organizado que los habitantes en otras regiones. Finalmente, los residentes en otros estados (resto del país), localizados en el centro del país, también han percibido al narcotráfico y el crimen organizado como una amenaza grave para el país, pero su opinión ha sido relativamente constante entre 2008 y $2012 .{ }^{37}$

\section{GRÁFICA 4}

Porcentaje que opina que el narcotráfico y el crimen organizado son una amenaza grave para el país, 2008, 2010 y 2012

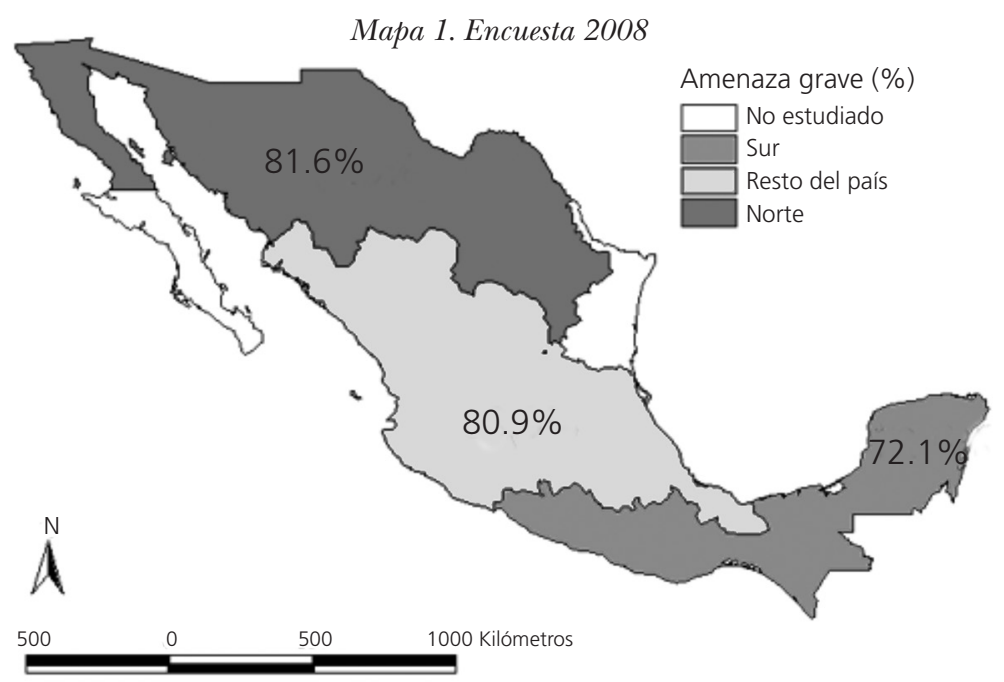

Hay que tomar algunas precauciones en la lectura de estos estimados muestrales. No nada más las diferencias regionales en estas proporciones (que son una cosa diferente a las medias aritméticas o

${ }^{37}$ En la frontera con la significancia estadística $(p=0.102)$ 

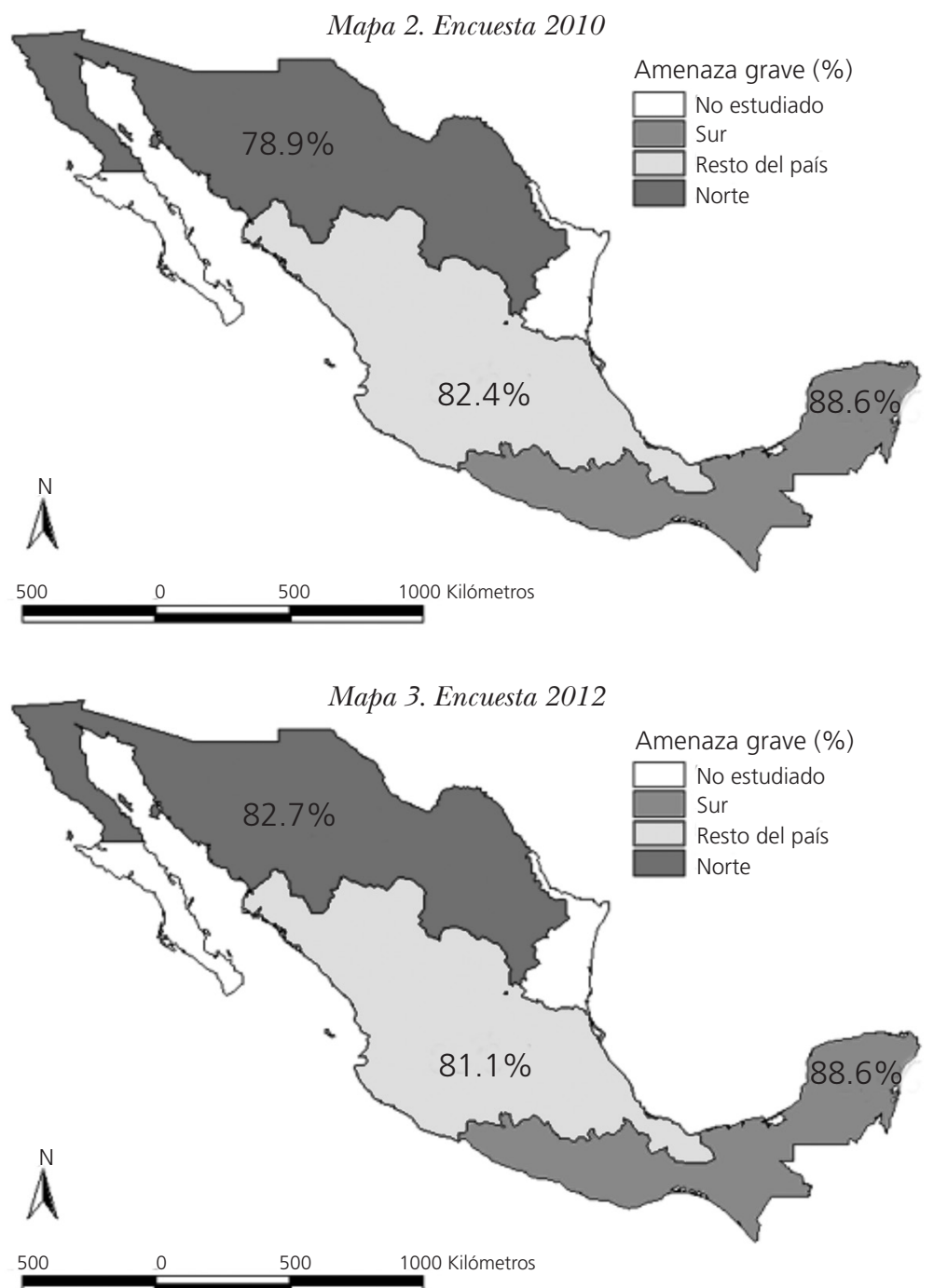

Fuente: elaboración propia con datos de la Encuesta México y el Mundo, 2008, 2012 y 2012.

los rangos promedio de la VD) pueden estar dentro del margen de error de la encuesta, sino que, como se verá en la sección siguiente, en la consideración de otros correlativos, estas diferencias regionales 
pueden modificarse. Lo que agrega una mayor complejidad a la prueba de hipótesis y a la comprensión del efecto contextual local.

\subsection{Los correlativos de la percepción}

Ya se dijo que la hipótesis nula de este estudio es que el contexto local, en este caso regional, no guarda relación con la percepción de la gravedad del problema del narcotráfico y el crimen organizado como una amenaza grave para el país. Los resultados del análisis multivariado sugieren rechazar esta hipótesis.

En detalle, los resultados del análisis multivariado muestran que, en 2008, 2010 y 2012, la opinión de la población fue significativamente diferente entre regiones, aun considerando la covariación de las variables composicionales o de control en este caso. Es decir, sí hay indicios, al menos en un nivel descriptivo y correlacional, de que el contexto regional puede influir en la formación de la opinión pública, al menos en este tema.

Además del contexto local o regional, se detectó que las siguientes variables composicionales mantuvieron una correlación independiente con la opinión: género, grupo de edad, nivel de ingreso y acceso a telefonía fija. No obstante, ninguna de estas variables mantuvo una relación estadísticamente significativa todos los años; solamente la variable género mostró una significancia estadística por dos años consecutivos, en 2008 y 2010.

En este punto hay entender lo que significan los signos de los coeficientes de las ecuaciones resultantes. ${ }^{38}$ En el análisis de regresión logística ordinal, los coeficientes son utilizados para estimar la proclividad individual a coincidir con el valor más alto de la escala de la vD, ${ }^{39}$ en este caso, la respuesta de que el narcotráfico y el crimen organizado "no es una amenaza". La proclividad individual se calcula de la siguiente manera:

${ }^{38}$ La técnica de regresión ordinal ofrece una ecuación para cada nivel de la VD, manteniendo las pendientes iguales en cada nivel, pero las constantes diferentes.

${ }^{39}$ En el paquete spss. 


\section{Cuadro 6}

Resultados del análisis de regresión ordinal

\begin{tabular}{|c|c|c|c|}
\hline & 2008 & 2010 & 2012 \\
\hline \multicolumn{4}{|l|}{ Variable dependiente: } \\
\hline Amenaza grave & $1.216 * * *$ & $1.446^{* * *}$ & $1.309 * * *$ \\
\hline Amenaza importante pero no muy grave & $2.916^{* * *}$ & $3.804 * * *$ & $2.294 * * *$ \\
\hline Amenaza poco importante & $4.920 * * *$ & $5.264 * * *$ & $3.224 * * *$ \\
\hline \multicolumn{4}{|l|}{ Variables independientes: } \\
\hline Región Norte & 0.034 & $0.319 * *$ & 0.051 \\
\hline Región Sur & $-0.462 * *$ & $-0.302 *$ & $0.298 * *$ \\
\hline \multicolumn{4}{|l|}{ Variables de control: } \\
\hline Sin escolaridad & 0.126 & -0.343 & 0.350 \\
\hline Escolaridad básica & 0.313 & -0.051 & 0.116 \\
\hline Escolaridad media & -0.096 & -0.154 & 0.068 \\
\hline Hombre & $0.188^{*}$ & $0.230 * *$ & -0.020 \\
\hline Les alcanza bien y pueden ahorrar & $-0.609 * *$ & -0.222 & 0.018 \\
\hline Les alcanza justo, sin grandes dificultades & -0.148 & 0.012 & 0.201 \\
\hline No les alcanza y tienen dificultades & -0.207 & 0.267 & 0.113 \\
\hline 18 a 29 años & 0.110 & -0.043 & -0.027 \\
\hline 30 a 39 años & -0.057 & -0.312 & $-0.442 * *$ \\
\hline 40 a 49 años & -0.081 & -0.055 & -0.173 \\
\hline 50 a 59 años & 0.035 & -0.233 & -0.221 \\
\hline Sí teléfono fijo en la vivienda & 0.055 & $-0.234^{*}$ & -0.007 \\
\hline Sí celular & -0.009 & -0.038 & 0.008 \\
\hline Sí usa internet & 0.304 & -0.063 & 0.125 \\
\hline \multicolumn{4}{|l|}{ Diagnósticos: } \\
\hline Chi-Cuadrado del modelo: & $35.360 * * *$ & $31.803^{* *}$ & $24.660^{*}$ \\
\hline Nagelkerke pseudo-R²: & 0.021 & 0.021 & 0.033 \\
\hline Chi-Cuadrado de líneas paralelas: & 25.246 & 32.742 & $630.064^{* * *}$ \\
\hline Función de relación: & Logit & Logit & Probit \\
\hline $\mathrm{n}=$ & 2,322 & 2,304 & 1,111 \\
\hline
\end{tabular}




\section{Cuadro 7}

Variables de referencia en el análisis de regresión

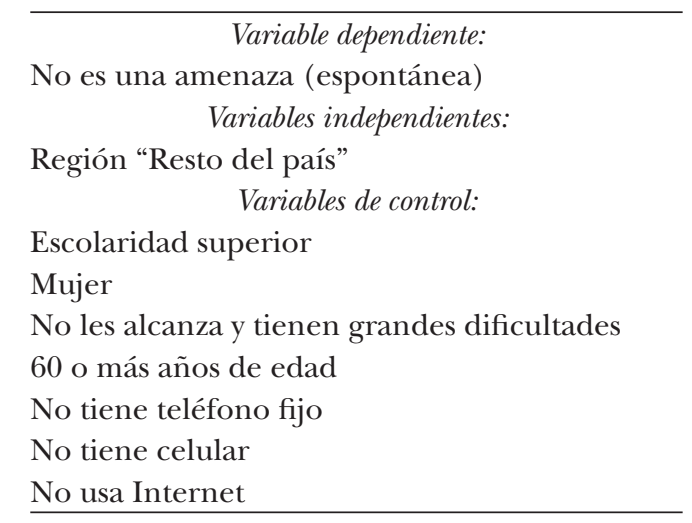

Fuente: cálculos propios.

De esta manera podemos saber que la proclividad de que un "hombre" coincida en opinar que el narcotráfico y el crimen organizado "no es una amenaza" es un $20.6 \%$ mayor $^{40}$ a la de una "mujer" de opinar lo mismo (véase el cuadro 8). ${ }^{41} \mathrm{~A}$ su vez, aquellos que tienen un ingreso que "les alcanza bien y pueden ahorrar" tienen una menor proclividad $(45.5 \% \text { menos })^{42}$ a opinar que el narcotráfico y el crimen organizado "no es una amenaza", frente a los demás sujetos que consideran que "no les alcanza y tienen grandes dificultades”. Esto sugiere que la composición de la población también sirve para predecir la opinión pública; es decir, no solamente el lugar o el contexto local pueden explicar todas las diferencias entre tales lugares o regiones en este caso. Pero recordemos que la capacidad explicativa de la composición no fue constante a lo largo

${ }^{40}$ Ya que $1.206-1=.206=20.6 \%$

${ }^{41}$ Una proclividad igual a 1 indicaría que no hay diferencia entre las diferentes categorías de la variable independiente o de control. Por este motivo se sustrae un valor de 1 de tal proclividad en los casos que rebase tal valor, o se sustrae un valor de 1 de tal proclividad en los casos en que sea menor de tal valor. Esto permite recalcular la proclividad a un porcentaje, lo que también facilita su interpretación.

${ }^{42}$ Ya que $1-0.545=0.455=45.5 \%$ 
de los años. Los anteriores correlativos composicionales no permiten predecir la opinión de forma consistente o todos los años. En cambio, la capacidad predictiva del contexto regional sí lo permite, aunque, notablemente, de forma cambiante en el periodo, como explicamos a continuación.

Para entender esta variación regional de la opinión, pasemos a revisar la magnitud del correlativo contextual entre 2008 y 2012. En este respecto, vemos que los habitantes de la región sur tenían una proclividad significativamente menor (-36.9\% en 2008 y $-26.0 \%$ en 2010) que los residentes de la región llamada "resto del país" a opinar que el narcotráfico y el crimen organizado "no es una amenaza". Es decir, independientemente de las variables composicionales en la ecuación, los habitantes del sur mantenían en tales años una percepción más grave del problema que los habitantes del centro. Esta opinión se vio significativamente modificada en 2012, en donde de hecho mostraron entonces una mayor proclividad a coincidir que los habitantes del centro del país, llamada región "resto del país" en la encuesta, a coincidir con la idea de que tal problema "no es una amenaza" para el conjunto del país. ${ }^{43}$

Finalmente, cabe advertirse que los coeficientes relacionados con las tres categorías de la variable dependiente (en relación con la categoría de referencia "no es una amenaza") son todos estadísticamente significativos. El valor de estas constantes en la línea de predicción de las probabilidades de que un encuestado se halle en uno u otro grupo de la variable dependiente debe incluirse en tal predicción. ${ }^{44}$

${ }^{43}$ Por lo anterior, la simple interpretación de los datos y mapas en frecuencias y medias aritméticas, o bien de una forma univariada o bivariada solamente, no es la correcta. En una prueba de diferencias de simples medias aritméticas podríamos erróneamente haber concluido exactamente lo contrario; no obstante, hecho el análisis multivariado, considerando la composición de las demás regiones en la ecuación, vemos que los hallazgos son más precisos. Lo anterior confirma la importancia de ambas perspectivas, composición y contexto local, pero siendo la segunda la que es consistente en el tiempo.

${ }^{44}$ Se unen a los coeficientes de las variables independientes para calcular las proclividades individuales de pertenecer a uno u otro grupo de la variable dependiente y manteniendo constante la variación de la categoría de referencia en la misma variable dependiente, en este caso, responder que el crimen organizado y el narcotráfico "no son una amenaza". 
CuAdro 8

Proclividades del modelo

\begin{tabular}{lccc}
\hline$\quad$ Variables independientes: & 2008 & 2010 & 2012 \\
\hline $\begin{array}{l}\text { Región Norte } \\
\text { Región Sur }\end{array} \quad 0.631$ & 0.740 & 1.346 \\
$\quad$ Variables de control: & & & \\
Hombre & 1.206 & 1.258 & \\
Les alcanza bien y pueden ahorrar & 0.545 & & \\
30 a 39 años & & & 0.644 \\
Sí teléfono fijo en la vivienda & & 0.792 & \\
\hline
\end{tabular}

Fuente: cálculos propios.

Nota: solamente se muestran las proclividades de aquellas variables que resultaron ser estadísticamente significativas.

\section{Discusión}

Los resultados de este estudio muestran evidencias de que es el contexto local, o regional en este caso, la única variable que predice la variación en la percepción de la amenaza del crimen organizado y el narcotráfico en México entre 2008 y 2012. Asimismo, se muestra evidencia de que las regiones mexicanas no parecen ser una división territorial sólo funcional y carente de un significado cultural, sino que parecen ser una división territorial con significados en las poblaciones que las habitan. Las variables composicionales, ni predijeron con más fuerza, ni con más consistencia temporal que las variables contextuales regionales, la percepción de la amenaza del crimen organizado.

Este estudio no termina de resolver el debate al respecto de si la geografía importa o no en la conformación de la percepción sobre este problema de seguridad pública; se necesita realizar más estudios con más controles composicionales y temporales. También se necesitan más estudios comparativos en el mismo objeto de estudio, y que es la percepción de gravedad del narcotráfico y crimen organizado en otros países que también atraviesan o han 
atravesado periodos históricos, igual o similarmente problemáticos, a los que atravesamos en México.

Este estudio enfrenta varias limitaciones. Primero y más claramente, no se pudo analizar a todos los estados del país a razón de las variaciones de inclusión o exclusión de los estados en cada vuelta de la encuesta; por este mismo motivo no se pudo incluir en el estudio los resultados de las vueltas de 2004 y 2006, los cuales habrían sido particularmente interesantes dado que la guerra contra el crimen organizado aún no había iniciado. No podemos, así, hacer una comparación antes/después de la percepción al respecto de este problema.

Segundo, además existe la posibilidad de que los estratos regionales definidos en la encuesta no necesariamente representen un lugar como tal en la mente de los encuestados; es decir, puede caber la posibilidad de que los estratos sean artefactos estadísticos elegidos sin mayor reflexión en el diseño de la encuesta, pero sin representar realmente lo que dicen representar. Por ejemplo: ¿qué significa la región "resto del país" frente a las otras dos opciones? En pocas palabras: tal vez estos estratos regionales no son la mejor opción de regionalización para poder someter a mayor prueba la hipótesis sobre la que gira el estudio.

En tercer lugar, también desconocemos el posible sesgo que la "no respuesta" a las preguntas de narcotráfico y crimen organizado haya tenido en los estimados regionales, siendo que este tema es particularmente delicado en ciertos lugares. Más allá de lo anterior, inclusive, desconocemos la tasa de rechazo de la encuesta en general y si ésta es función de la región de residencia del encuestado. La carga de la prueba, en este caso, correspondería primero a los encuestadores y luego a los analistas en la prueba de sus hipótesis respectivas.

Para finalizar, queda claro que no se podría hablar, al menos con la evidencia disponible, de una opinión nacional uniforme a lo largo del país. No tenemos evidencia de que México, entre 2008 y 2012, fuera un bloque de opinión homogéneo al respecto de la amenaza que significaban el narcotráfico y el crimen organizado. Más bien al contrario: cada quien opinó parcialmente en función de dónde vivía en ese periodo. Y fue la variable regional, aún con las 
limitaciones previamente descritas, la que mejor predijo estas mismas variaciones; ninguna variable composicional pudo arrebatarle, en ninguna vuelta de la encuesta, la superioridad de la correlación en este estudio. Esto significa, en términos de política pública, que las políticas nacionales, y en este caso las políticas de seguridad pública federales, no serán necesariamente las preferidas en todas las regiones que constituyen a la nación o federación. Dicho de otra manera, los llamados intereses nacionales pueden diferir de los regionales y viceversa. Y esto podría, tal vez, condicionar las posibilidades de éxito de las políticas en los dos ámbitos de gobierno.

\section{Bibliografía}

Agnew, John A., Place and Politics: The Geographical Mediation of State and Society, Londres, Allen and Unwin, 1987.

, "The Territorial Trap: The Geographical Assumptions of International Relations Theory", Review of International Political Economy, vol. 1, 1994, pp. 53-80.

- "Mapping Politics: How Context Counts in Electoral Geography”, Political Geography, vol. 15, núm. 2, 1996, pp. 129-146.

Elden, Stuart, "Land, Terrain, Territory", Progress in Human Geography, vol. 34, 2010, pp. 799-817.

Ethington, P. y J. McDaniel, "Political Places and Institutional Spaces: The Intersection of Political Science and Political Geography", Annual Review of Political Science, vol. 10, 2007, pp. 127-142.

Fisher, R. A., The Design of Experiments, Edimburgo, Oliver \& Boyd, 1935.

Flint, Colin, "Forming Electorates, Forging Spaces: The Nazi Party Vote and the Social Construction of Space", American Behavioral Scientist, vol. 41, núm. 9, 1998, pp. 1282-1303.

— M. Harrower y R. Edsall, But How Does Place Matter? Using Bayesian Networks to Explore a Structural Definition of Place, documento presentado en la New Methodologies for the Social Sciences Conference, University of Colorado at Boulder, 2000.

Giddens, Anthony, "Agency, Institution, and Time-Space Analysis", en Karin Knorr-Cetina y Aaron. V. Cicourel (eds.), Advances in Social Theory 
and Methodology. Toward An Integration of Micro- and Macro-Sociologies, Boston, Routledge \& Kegan Paul, 1981.

- The Constitution of Society, Berkeley, University of California Press, 1984.

- The Consequences of Modernity, Stanford, Standford University Press, 1990.

$-\mathrm{y}$ Christopher Pierson, Conversations with Anthony Giddens. Making Sense of Modernity, Cambridge, Polity Press, 1998.

Gieryn, Thomas F., "A Space for Place in Sociology", Annual Review of Sociology, vol. 26, 2000, pp. 463-496.

Huckfeldt, Robert y John Sprague, "Political Parties and Electoral Mobilization: Political Structure, Social Structure, and the Party Canvass", American Political Science Review, marzo de 1992, pp. 70-86.

King, G., "Why Context Should Not Count", Political Geography, vol. 15, núm. 2, 1996, 159-164.

Lutz, J., "Diffusion of Voting Support: the Radical Party in Italy", en M. Eagles (ed.), Spatial and Contextual Models in Political Research, Londres, Taylor and Francis, 1995.

Magaloni, B., A. Díaz-Cayeros y V. Romero, "La raíz del miedo: ¿Por qué es la percepción de riesgo mucho más grande que las tasas de victimización?, en José Antonio Aguilar (coord.), Las bases sociales del crimen organizado, México, Secretaría de Seguridad Pública (SSP) y Centro de Investigación en Estudios en Seguridad (CIES), 2012, capítulo 4.

Massey, Doreen B., Space, Place, and Gender, Minneapolis, University of Minnesota Press, 1994.

McAllister, Ian y Donley Studlar, "Region and Voting in Britain, 1979-87: Territorial Polarization or Artifact?”, American Journal of Political Science, vol. 36, núm. 1, 1992, 168-199.

O’Loughlin, J., "Spatial Analysis in Political Geography”, en J. Agnew, K. Mitchell y G. Tuathail (eds.), A Companion to Political Geography, Oxford, Basil Blackwell, 2002.

Pred, A., "Context and Bodies in Flux: Some Comments on Space and Time in the Writings of Anthony Giddens", en J. Clark, C. Modgil y S. Modgil (eds.), Anthony Giddens: Consensus and Controversy, Londres, The Falmer Press, 1990, pp. 117-129.

Sauerzopf, Richard y Todd Swanstrom, "The Urban Electorate in Presidential. Elections, 1920-1996”, Urban Affairs Review, vol. 35, 1999, pp. 72-91. 
Shin, Michael E. y John A. Agnew, "The Geography of Party Replacement in Italy, 1987-1996", Political Geography, vol. 21, 2002, pp. 221-242.

Tobler, Waldo R., "A Computer Movie Simulation Urban Growth in the Detroit Region”, Economic Geography, vol. 46, núm. 2, 1970, 234-240.

Vilalta, Carlos J., "Perspectivas geográficas en la sociología urbana: La difusión espacial de las preferencias electorales y la importancia del contexto local”, Estudios Demográficos y Urbanos, vol. 18, núm. 3, 2003, 147-177.

, "The Local Context and the Spatial Diffusion of Multiparty Competition in Urban Mexico, 1994-2000”, Political Geography, vol. 23, núm. 4, 2004, pp. 403-423.

-, "How Exactly Does Place Matter in Crime Analysis? Place, Space and Spatial Heterogeneity", Journal of Criminal Justice Education, vol. 24, núm. 3, 2013.

-, "Does the Mexican War on Organized Crime Mediate the Impact of Fear of Crime on Daily Routines?”, Crime Eं Delinquency, 2014. DOI: $10.1177 / 0011128714541208$. 